CLAWAR 2020: 23rd International Conference on

Climbing and Walking Robots and the Support

Technologies for Mobile Machines,

Moscow, Russian Federation, 24-26 August 2020.

https://doi.org/10.13180/clawar.2020.24-26.08.16

\title{
Polyhedral Rolling Robot with Expanded Icosidodecahedron Body
}

\author{
Mizuho Shibata and Yushi Azuma \\ Department of Robotics, Kindai University, \\ 1 Takaya-Umenobe, Higashi-hiroshima, Hiroshima 739-2116, Japan \\ E-mail: shibata@hiro.kindai.ac.jp
}

\begin{abstract}
This manuscript describes the rolling locomotion of a polyhedral robot. Polyhedral robots may be able to be stationary without wasting energy because they contact with a slope at a surface or multiple points. However, knowledge of the mechanisms and the control strategies of the polyhedron robot is little. The outer shell of our prototype has an expanded icosidodecahedron body, which has four types of contact surfaces. This robot has an eccentric motor inside to shift the center of gravity of the robot and generate the inertia force. This robot rolls by the reaction force of the centripetal force when the eccentric motor is rotated. In this manuscript, a rolling condition of a polyhedral robot is derived based on Zero Moment Point (ZMP). Based on several analyses, we show that changing the magnitude of the angular velocity of the motor shifts the position of the ZMP; however, the angular velocity should be set an appropriate value. Through several experiments, we clarify the characteristics of this prototype.
\end{abstract}

\section{Introduction}

This manuscript discusses the rolling motion of a polyhedral robot. In the field of robotics, various mobile robots have been studied. In particular, spherical robots are expected to play an active role in various areas of the leveled floor. In spherical robots, many types of research have been conducted not only from the viewpoint of mechanisms but also from the viewpoint of driving strategies. In the researches of the mechanisms, the designs of the internal structure are briefly studied, and driving mechanisms with a simple wheel, ${ }^{1}$ a car, ${ }^{2}$ fixed internal propulsion mechanisms, ${ }^{3}$ attached rotors, ${ }^{4}$ gyros ${ }^{5}$ and eccentric motors ${ }^{6}$ have been proposed. In the researches of the driving strategies, the studies in terms of feedback control, ${ }^{7}$ stability analysis, ${ }^{8}$ and motion planning ${ }^{9}$ have been proposed.

In comparison with the researches of the spherical robot, the research of the polyhedral robot is very little from the viewpoint of the mechanisms and the driving strategies. A polyhedral robot with a tensegrity body ${ }^{10}$ has been proposed as an outer shell mechanism. In the research of an internal mechanism of a polyhedral robot, a cubic robot with inverted pendulums $^{11}$ has been proposed. Since spherical robots make contact with a slope at one point, they must consume energy when they are stationary. On the other hand, polyhedral robots may be able to be stationary without wasting energy because they contact with a slope at a surface or multiple points. Moreover, the fundamental conditions for the continuous rolling of a polyhedral robot have not been clarified.

This manuscript reveals a rolling principle of a polyhedral robot with eccentric motors. The conditions under which a polyhedral robot rolls when a motor performs uniform circular motion are examined through a physical model. A rolling condition of a polyhedral robot is derived based on Zero Moment Point (ZMP). This manuscript is organized as follows: the next section briefly outlines the fabrication and the formulation of a polyhedral robot with eccentric motors. An expanded icosidodecahedron shape is applied to the outer shell. Section III discusses experimental results to reveal the fundamental properties of our prototype. We confirm the rolling angle of a polyhedral robot for a slope. We experimentally evaluate that a polyhedral robot can be stationary on a slope without energy consumption. We also experimentally confirm that the polyhedral robot rolls by the uniform circular motion of 


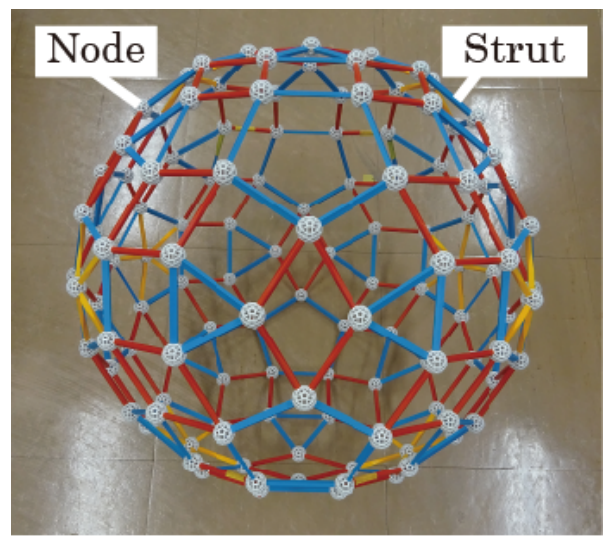

Fig. 1. Outer shell

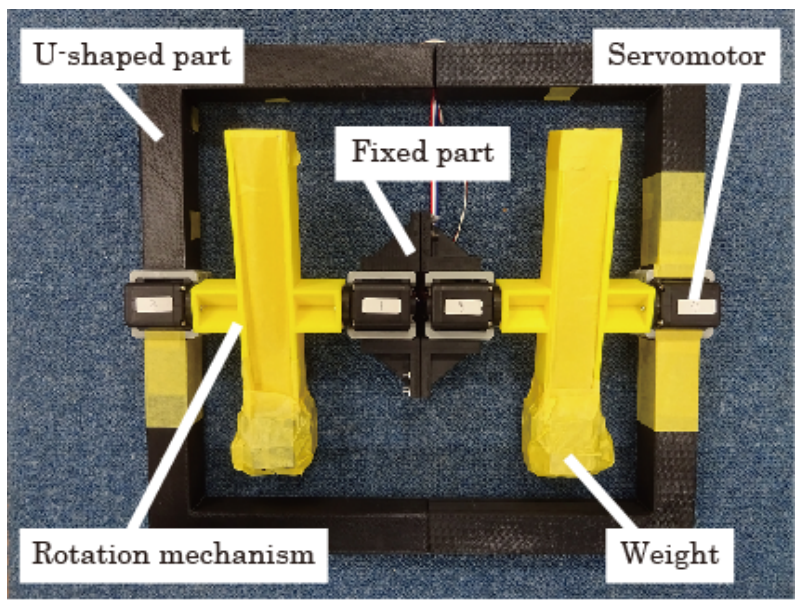

Fig. 2. Rotation mechanism

the eccentric motor. Section IV presents our summary.

\section{Expanded icosidodecahedron-type rolling robot}

\subsection{Outer shell}

This section describes the outer shell of the polyhedral robot developed in this research. A toy called "Zometool" 12 is applied to the outer shell. Zometool is composed of spherical parts called nodes and rod parts called struts. The diameter of the node is approximately 18 [mm]. Our prototype consists of three kinds of struts, blue, red, and yellow struts. The lengths of blue, red and yellow struts are $65[\mathrm{~mm}], 62[\mathrm{~mm}]$ and $55[\mathrm{~mm}]$, respectively. This prototype applies an extended icosidodecahedron body to the outer shell (Figure 1). The body consists of 120 red struts, 120 blue struts, and 120 nodes. The expanded icosidodecahedron body consists of four-type surfaces: triangle, quadrangle, pentagon, and rhombus. These surfaces are 20 triangles, 60 quadrangles, 12 pentagons, and 30 rhombuses. Besides, 40 yellow struts and eight nodes are used to add to the strength of the pentagonal surface of the outer shell. The outer shell has a diameter of about 470 [mm], the circumference of about 1477 [mm], and the mass of $437[\mathrm{~g}]$. Since this robot has four kinds of surfaces, the rolling from various contact situations between the body and the floor can be confirmed experimentally. 


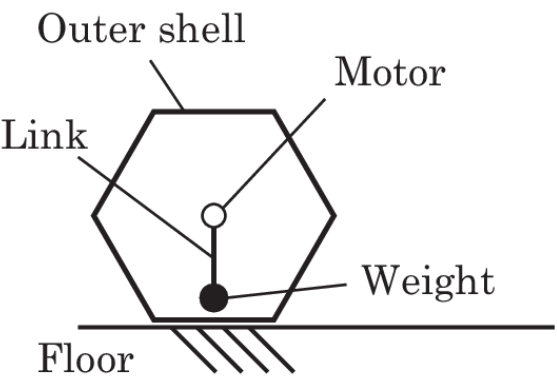

(a) Initial state

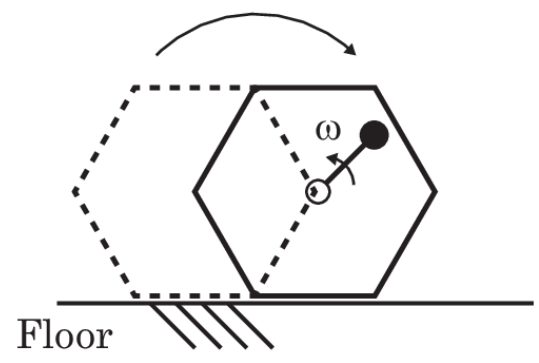

(b) Rolling state

Fig. 3. Rolling concept

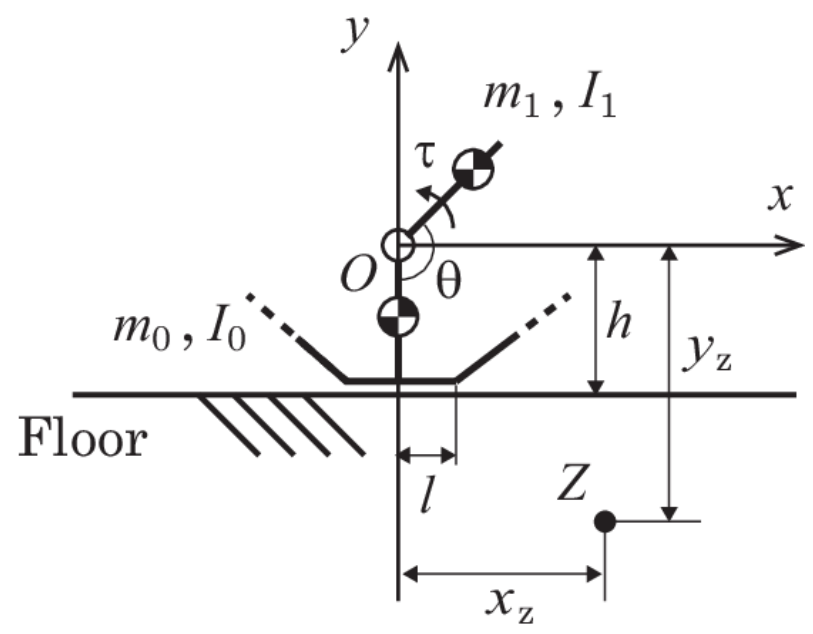

Fig. 4. Physical model of a polyhedral rolling robot with an eccentric motor

\subsection{Internal mechanism}

Figure 2 shows the internal mechanism of the polyhedral robot. The mechanism consists of two eccentric motors. A link with a weight at the tip is fixed to each motor. The rotation of these motors changes the gravity offset and rotational inertia of the polyhedral robot. The rotational mechanisms with a height of 184 [mm], a width of 80 [mm], and a depth of 26 [mm] were fabricated by a 3D printer using ABS resin. Two servomotors (Dynamix AX$18 \mathrm{~A}$, made by ROBOTIS) were fixed to both sides of the rotation mechanism. The weights are 200 [g]; hence, the total weight of 400 [g]. In order, U-shaped parts are fixed the outer motors. This U-shaped part is fixed to the outer shell to prevent the motor from spinning around. The mass of the U-shaped mechanism is 940 [g], and the total weight of the internal mechanism is $1340[\mathrm{~g}]$. By moving two motors simultaneously, the robot realizes a linear rolling motion.

\subsection{Rolling principle}

This robot rotates using the gravity offset and rotational inertia of the internal mechanism. As stated in Figure 2, the internal mechanism consists of the link with weights at the tip. In the initial state, the link is positioned vertically downward and rotated toward the robot's traveling direction (Figure 3). Figure 4 shows the model of the robot to clarify the rolling 
principle. This model verifies the rolling motion in the 2 -dimensional plane with gravity. The position of the rotary motor is the origin of the coordinates. Let $\tau$ be the torque applied to the motor. As shown in the figure, $\theta$ is the angle between the link and the y-axis. Let $l$ be the distance between the edge against the moving direction of the outer shell and the $y$-axis. One surface of the outer shell of the polyhedral robot contacts on the flat floor. Let $h$ be the distance between the ground and the axis of rotation. Let $m_{0}$ and $I_{0}$ be the mass and the moment of inertia of the outer shell. Let $m_{1}$ and $I_{1}$ be the mass and the moment of inertia of the link with a weight. Henceforth, the subscripts 0 and 1 imply the outer shell and the link, respectively. Let $\omega_{i}(i=0,1)$ be the angular velocity of the shell and the link. The positions of the center of gravity of the shell and the mass are also described as $x_{i}$ and $y_{i}(i=0,1)$.

Zero Moment Point (ZMP) ${ }^{13}$ is used to formulate the rolling condition of polyhedral robots. The ZMP is the center of pressure of the floor reaction force, and when the ZMP exists outside the support polygon of the robot, the polyhedral robot rotates. Many studies of ZMP have been carried out from the viewpoint of gait generation of biped robots. ${ }^{14-16}$ We set point $Z$ inside the floor and calculate the moment around point $Z$. Let $g$ be the gravitational acceleration. The moment around the point $Z, M_{Z}$, describes the sum of the moment of inertia and the moment of gravity. Therefore, $M_{Z}$ is described as the following equation.

$$
M_{Z}=\sum_{i=0}^{1} I_{i} \dot{\omega}_{i}+\sum_{i=0}^{1} m_{i}\left\{\ddot{x}_{i}\left(y_{\mathrm{Z}}-y_{i}\right)-\ddot{y}_{i}\left(x_{\mathrm{Z}}-x_{i}\right)\right\}+\sum_{i=0}^{1} m_{i}\left(x_{\mathrm{Z}}-x_{i}\right) g,
$$

where $x_{\mathrm{Z}}$ and $y_{\mathrm{Z}}$ are the $x$ and $y$ positions of the point $Z$, respectively. Assuming that the $\mathrm{ZMP}\left(x_{\mathrm{Zmp}},-h\right)$ is located on the floor, Equation $(1)$ becomes as follows.

$$
0=\sum_{i=0}^{1} I_{i} \dot{\omega}_{i}+\sum_{i=0}^{1} m_{i}\left\{-\ddot{x}_{i}\left(h+y_{i}\right)-\ddot{y}_{i}\left(x_{\mathrm{Zmp}}-x_{i}\right)\right\}+\sum_{i=0}^{1} m_{i}\left(x_{\mathrm{Zmp}}-x_{i}\right) g .
$$

Equation (2) rearranges for $x_{\mathrm{zmp}}$ :

$$
x_{\mathrm{Zmp}}=\frac{m_{1} \ddot{x}_{1}\left(h+y_{1}\right)-m_{1} x_{1} \ddot{y}_{1}+m_{1} x_{1} g}{\left(m_{0}+m_{1}\right) g-m_{1} \ddot{y}_{1}},
$$

where $\dot{\omega}_{0}, \ddot{x}_{0}$ and $\ddot{y}_{0}$ are zero. We assumed that the center of gravity of the outer shell coincides with the origin $\left(x_{0}=0\right.$ and $\left.y_{0}=0\right)$. For the sake of simplicity, we also assumed that the link is an uniform circular motion $\left(\dot{\omega}_{1}=0\right)$.

To clarify the structure of the analytical equation, the ZMP is confirmed when the link is in a uniform circular motion. The magnitude of the angular velocity of the link in this situation is assumed to be $w$; hence, $w_{1}=w$. The distance between the center of gravity of the link and the axis of rotation denotes $r_{1}$, and the following equations are satisfied.

$$
\begin{gathered}
\ddot{x}_{1}=r_{1} \omega^{2} \sin \theta, \\
\ddot{y}_{1}=-r_{1} \omega^{2} \cos \theta .
\end{gathered}
$$

Therefore, Equation (3) describes as follows:

$$
x_{\mathrm{Zmp}}(\theta)=\frac{\left(h m_{1} r_{1} \omega^{2}+m_{1} r_{1} g\right) \sin \theta}{\left(m_{0}+m_{1}\right) g+m_{1} r_{1} \omega^{2} \cos \theta} .
$$

The ZMP is expressed as a function of angle $\theta$. Here, $\max \left\{x_{\mathrm{Zmp}}(\theta)\right\}$ is defined as $x_{\text {max }}$ when $0<\theta<\pi$, and then, the conditions under which the polyhedral robot rolls are expressed by the following equation.

$$
x_{\max }>l .
$$




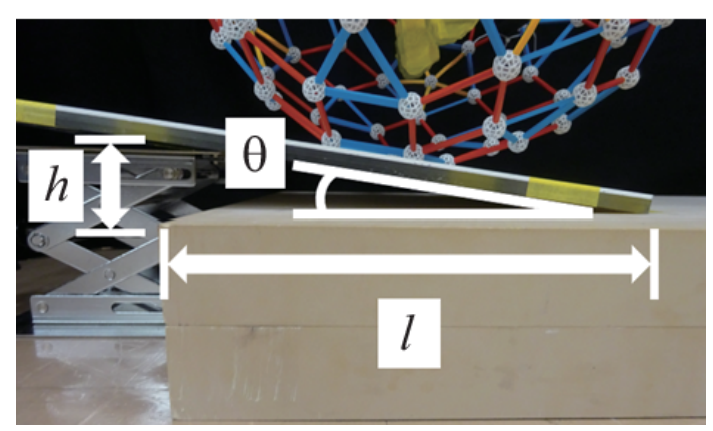

Fig. 5. Experimental setup for measuring rolling angles

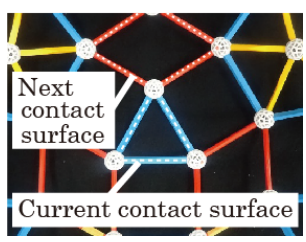

(a)

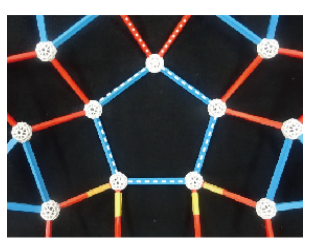

(e)

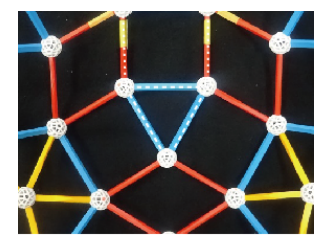

(b)

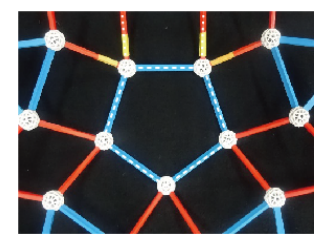

(f)

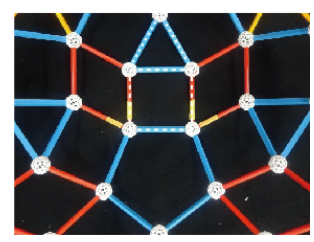

(c)

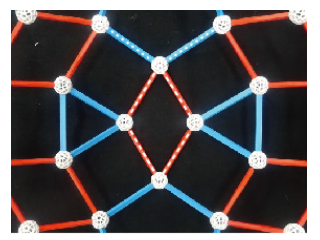

(g)

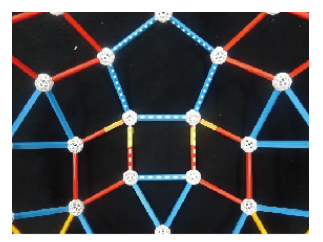

(d)

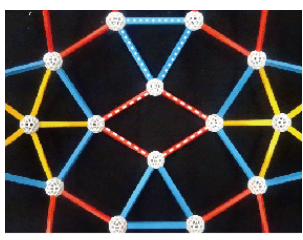

(h)

Fig. 6. Contact situations

We have $x_{\max }$ as follows:

$$
x_{\max }=\frac{h m_{1} r_{1} \omega^{2}+m_{1} r_{1} g}{\sqrt{\left(m_{0}+m_{1}\right)^{2} g^{2}-m_{1}^{2} r_{1}^{2} \omega^{4}}},
$$

where, $x_{\max }$ is strictly increasing with respect to $\omega$. Therefore, changing the magnitude of $\omega$ shifts the position of the ZMP; however, the angular velocity $\omega$ should be satisfying the following condition:

$$
\left(m_{0}+m_{1}\right) g>m_{1} r_{1} \omega^{2}
$$

\section{Experiments}

\subsection{Rolling angle of each contact situation}

In this manuscript, the maximum angle of the slope where the polyhedral robot does not roll is called "rolling angle." The experimental setup for measuring rolling angles shows Figure 5 . We set the base $l$ of the slope to $300[\mathrm{~mm}]$, and change the height $h[\mathrm{~mm}]$ to calculate the angle $\theta[\mathrm{rad}]$ based on the following equation:

$$
\theta=\tan \left(\frac{h}{l}\right) \times \frac{\pi}{180}
$$

The surface of the slope is composed of a resin board (PLAPLATE 2mm B4SIZE made by Tamiya). We measure five times the rolling angle for a contact condition and calculate the average value. The robot is not energized during the experiments. 
In these measurements, not only the shape of the current contacting surface but also the traveling direction are considered. That is, the shape of the next contacting surface after rolling is also considered. In our prototype that has an expanded icosidodecahedron body, the contacting situations are eight patterns: (a) transition from a triangular surface to a rhombus surface, (b) transition from a triangular surface to a quadrangular surface, (c) transition from a quadrangular surface to a triangular surface, (d) transition from a quadrangular surface to a pentagonal surface, (e) transition from a pentagonal surface to a rhombus surface, (f) transition from a pentagonal surface to a quadrangular surface, (g) transition from a rhombus surface to a pentagonal surface, $(\mathrm{h})$ transition from a rhombus surface to a triangular surface. The vertices of our prototype are composed of nodes; therefore, the robot is in multi-point contact with the floor.

Table 1 shows the rolling angle for each contacting situation. From the table, the polyhedral robot could be stationary without wasting energy because they contact with a slope at multiple points. Moreover, it was clarified that the rolling easiness of this prototype was different for each contacting surface and traveling direction. In our prototype, the case of (c) is the contact state in which the robot rotates most easily. These experiments are consistent with placing the ZMP of the robot outside the support polygon of the outer shell by tilting the slope. According to Equation (7), the rolling condition depends on the distance between the axis of rotation and the edge of the outer shell. Therefore, the reason why the rolling angle differs according to the traveling direction even in the same contacting surface is due to the difference of length $l$.

\subsection{Rolling motion}

This section describes the effect of the angular velocity of the motor of the internal mechanism on the translational speed of the prototype. As stated in the previous section, the rolling easiness of this prototype was different for each contacting surface and traveling direction. In this experiment, the rolling from the easiest situation (case (c)) was applied to the initial state of the prototype. Based on Equation (9), we choose the angular velocity $\omega$ carefully. In our prototype, $m_{0}, m_{1}, r$ and $g$ were $1.29[\mathrm{~kg}], 0.51[\mathrm{~kg}], 0.085[\mathrm{~m}]$ and $9.81\left[\mathrm{~m} / \mathrm{s}^{2}\right]$, respectively. Therefore, we should set $\omega$ below $20.2[\mathrm{rad} / \mathrm{s}]$. The magnitude of the translational velocity is measured when the robot is placed on a flat floor, and the travel distance is $2[\mathrm{~m}]$. Figure 7 shows a typical rolling motion. In this figure, the angular velocity $\omega$ is $3.8[\mathrm{rad} / \mathrm{s}]$, and the polyhedral robot rolls by the uniform circular motion of the eccentric motor.

Figure 8 shows the relationship between the angular velocity of the servomotor and the translational speed of the body. The angular velocity of the internal servomotor was varied in the range of $1.7-4.5[\mathrm{rad} / \mathrm{s}]$. When the angular velocity was set outside this range, the body did not move sufficiently, and the data could not be measured. From the result of Figure 8, it was found that the translational speed of the body was largest when the angular velocity of the motor was $3.8[\mathrm{rad} / \mathrm{s}]$ in this prototype. Therefore, these results suggest that the angular velocity of the motor of the internal mechanism must be set appropriately to increase the translational speed. As stated in Equation (6), the position of ZMP depends on the link angle $\theta$. That is, it is suggested that the angle $\theta$ should be kept constant during the motion of the robot to keep the position of ZMP outside the support polygon of the outer shell and continue rolling at a constant translational speed. This is a future work to be solved from analyses and experiments. 
Table 1. Rolling angles against each contact situation

\begin{tabular}{lllll}
\hline \multicolumn{5}{c}{ Contact surface } \\
& Current & Next & $h[\mathrm{~mm}]$ & $\theta[\mathrm{rad}]$ \\
\hline \hline (a) & Triangle & Rhombus & 55.0 & 0.181 \\
\hline (b) & Triangle & Quadrangle & 36.8 & 0.122 \\
\hline (c) & Quadrangle & Triangle & 24.4 & 0.081 \\
\hline (d) & Quadrangle & Pentagon & 72.2 & 0.236 \\
\hline (e) & Pentagon & Rhombus & 51.6 & 0.170 \\
\hline (f) & Pentagon & Quadrangle & 79.2 & 0.258 \\
\hline (g) & Rhombus & Pentagon & 70.0 & 0.229 \\
\hline (h) & Rhombus & Triangle & 26.6 & 0.088 \\
\hline
\end{tabular}

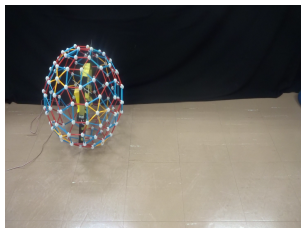

(a) $0 \mathrm{~s}$

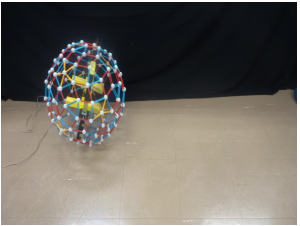

(b) $1 \mathrm{~s}$

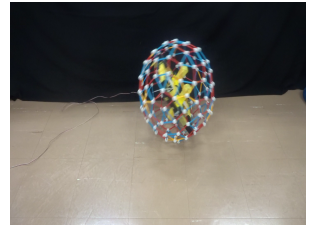

(c) $2 \mathrm{~s}$

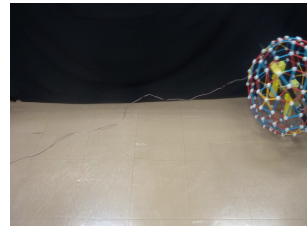

(d) $3 \mathrm{~s}$

Fig. 7. Typical rolling motion $(\omega=3.8[\mathrm{rad} / \mathrm{s}])$

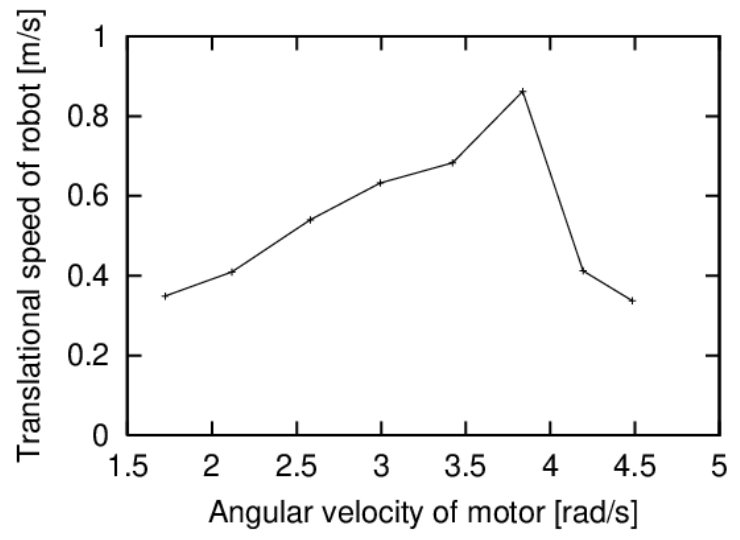

Fig. 8. Experimental results (weight: 400 [g])

\section{Summary}

This paper described a rolling principle of a polyhedral robot with eccentric motors. We applied an expanded icosidodecahedron body to the outer shell of the prototype because the body has four kinds of surfaces. Polyhedral robots could be stationary without wasting energy because they contact with a slope at multiple points. This robot rotated using the gravity offset and rotational inertia of the internal mechanism through the physical model. Notably, the conditions under which a polyhedral robot rolls when a motor performs uniform circular motion were examined through a physical model.

A rolling condition of a polyhedral robot was derived based on Zero Moment Point (ZMP). Through analyses, changing the magnitude of the angular velocity of the motor shifts the position of the ZMP; however, the angular velocity should be set an adequate value. Moreover, We evaluated the relationship between the angular velocity of the motor of the internal mechanism and the translational speed of the body experimentally. The results indicated that the angular velocity of the motor of the internal mechanism had to 
be appropriately set to raise the moving speed in this prototype.

In this manuscript, only linear motion is considered; hence, the future works include the realization of three-dimensional movement. Besides, the effect of angular acceleration was not verified in detail. According to Equation (2), the position of the ZMP depends on the moment of inertia and angular acceleration of the prototype. There is a possibility that the ZMP is easily placed outside the supporting polygon of the outer shell by the starting torque, and it should be examined analytically and experimentally in the future. Regarding the effect of angular acceleration, the magnitude of the floor reaction force should be examined.

\section{References}

1. A. Halme, T. Schonberg and Y. Wang, "Motion control of a spherical mobile robot," Proceedings of IEEE International Workshop on Advanced Motion Control, pp. 259-264, 1996.

2. A. Bicchi, A. Balluchi, D. Prattichizzo and A. Grelli, "Introducing the Sphericle: An Experimental Testbed for Research and Teaching in Nonholonomy," Proceedings of IEEE International Conference on Robotics and Automation, pp. 2620-2625, 1997.

3. S. Bhattacharya and S. K. Agrawal, "Spherical Rolling Robot: A Design and Motion Planning Studies," IEEE Transaction on Robotics and Automation, Vol. 16, No. 6, pp. 835-839, 2000.

4. A. H. Javadi A and P. Mojabi, "Introducing August: a novel strategy for an omnidirectional spherical rolling robot," Proceedings IEEE International Conference on Robotics and Automation, pp. 3527-3533, 2002.

5. T. Otani, T. Urakubo, S. Maekawa, H. Tamaki and Y. Tada, "Position and attitude control of a spherical rolling robot equipped with a gyro," Proceedings of IEEE International Workshop on Advanced Motion Control, pp. 416-421, 2006.

6. M. Ishikawa, R. Kitayoshi and T. Sugie, "Volvot : A spherical mobile robot with eccentric twin rotors," Proceedings of IEEE International Conference on Robotics and Biomimetics, pp. 1462-1467, 2011.

7. T. Yamanaka, S. Nakaura and M. Sampei, "Hopping Motion Analysis of Superball-like Spherical Robot Based on Feedback Control," Proceedings of IEEE/RSJ International Conference on Intelligent Robots and Systems, pp. 3805-3810, 2003.

8. V. Muralidharan and A. D. Mahindrakar, "Geometric Controllability and Stabilization of Spherical Robot Dynamics," IEEE Transactions on Automatic Control, Vol. 60, Issue 10, pp. 2762-2767, 2015.

9. V. Dwaracherla, S. Thakar, L. Vachhani, A. Gupta, A. Yadav and S. Modi, "Motion Planning for Point-to-Point Navigation of Spherical Robot Using Position Feedback," IEEE/ASME Transactions on Mechatronics, Vol. 24, Issue 5, pp. 2416-2426, 2019.

10. M. Shibata, F. Saijyo and S. Hirai, "Crawling by Body Deformation of Tensegrity Structure Robots," Proceedings of IEEE International Conference on Robotics and Automation, pp.43754380, 2009.

11. M. Gajamohan, M. Merz, I. Thommen and R. D'Andrea, "The Cubli: A Cube that can Jump Up and Balance," Proceedings of IEEE/RSJ International Conference on Intelligent Robots and Systems, pp. 3722-3727, 2012.

12. G. W. Hart and H. Picciotto, "Zome Geometry Hands-on Learning with Zome Models," Key Curriculum Press, 2001.

13. M. Vukobratović and J. Stepanenko, "On the Stability of Anthropomorphic Systems," Mathematical Biosciences, Vol. 15, pp.1-37, 1972.

14. A. Goswami, "Foot rotation indicator (FRI) point: a new gait planning tool to evaluate postural stability of biped robots," Proceedings of IEEE International Conference on Robotics and Automation, pp. 47-52, 1999.

15. S. Kajita, F. Kanehiro, K. Kaneko, K. Fujiwara, K. Harada, K. Yokoi and H. Hirukawa, "Biped walking pattern generation by using preview control of zero-moment point," Proceedings of IEEE International Conference on Robotics and Automation, pp. 1620-1626, 2003.

16. K. Mitobe, S. Kaneko, T. Oka, Y. Nasu and G. Capi, "Control of legged robots during the multi support phase based on the locally defined ZMP," Proceedings of IEEE/RSJ International Conference on Intelligent Robots and Systems, pp. 2253-2258, 2004. 